\title{
Association of Serum Asymmetric Dimethylarginine with the Severity of Coronary Artery Disease: A Pilot Study
}

\author{
Lakshmi Goudhaman $^{1}$, Anusha Raja Jagadeesan ${ }^{1}$, Saranya Sundaramoorthi ${ }^{1}$, \\ Spandana Thotla*1, Surapaneni Krishna Mohan ${ }^{1}$
}

\begin{abstract}
Background: Asymmetric dimethylarginine (ADMA), an inhibitor of nitric oxide synthase (NOS), has been implicated in endothelial dysfunction and atherogenesis. Though there is much evidence linking ADMA with atherosclerosis and adverse cardiovascular events, only a few studies have established the independent relationship between elevated ADMA and the angiographic extent of coronary artery disease (CAD). The aim of the study was to analyze serum ADMA levels in patients with varied extent and severity of coronary atherosclerosis and to see whether the levels of ADMA in male and female participants vary significantly.

Methods: We analyzed 40 individuals with obstructive CAD, including men and women, between the ages of 30 and 60 . According to their coronary angiographic reports, the participants were divided into four groups: minor CAD, single vessel disease (SVD), double vessel disease (DVD) group and triple vessel disease (TVD). Then, serum ADMA levels was measured and compared among these groups.

Results: ADMA level was significantly higher in patients with TVD $(167.74 \pm 16.69)$ than those in the DVD (159.46 \pm 10.40$)$, SVD (149.54 \pm 16.39$)$ and minor CAD (144.5 \pm 24.16$)$ group (p-value= 0.0001). There was no significant difference in ADMA levels between male and female participants $(\mathrm{p}=0.534)$.

Conclusions: ADMA concentration in the serum may be useful in identifying whether CAD correlates significantly to the extent and severity of coronary atherosclerosis.
\end{abstract}

Keywords: ADMA, CAD, Endothelial dysfunction, NOS, Atherosclerosis.

\section{Introduction}

Coronary artery disease (CAD) is the most common form of cardiovascular disease worldwide and leading cause of disability and death in both developed and developing countries $(1,2)$. CAD is primarily caused by atherosclerosis, which results in a regional decrease in myocardial blood flow (2). The inadequate blood and oxygen supply to the myocardium results in decreased myocardial blood flow and ischemia (2). Each year, the prevalence and global burden of CAD continues to rise, and as predicted, atherosclerosis is now the leading global cause of total disease burden (3). Despite the development of medical interventions to minimize fatal CAD outcomes, the mortality rate remains high (4). In developed countries, the CAD rate increased by $30-60 \%$ between 1990 to 2020, while it increased even more in developing countries (4). In addition to having a higher mortality rate, CAD manifested 10 years earlier in India compared to the rest of the world. As a result, there were more CAD-related deaths among India's working-age population (5). Due to CAD related deaths, India lost a significant amount of productive working years. By 2030, it 
is estimated that 17.9 million productive working years will be lost, which is 10 times higher than the United States $(6,7)$.

Asymmetric dimethylarginine (ADMA) was found to be a novel risk marker of atherosclerosis. It is considered an independent risk marker and a prognostic marker of CAD. Asymmetric dimethylarginine originates from the degradation of methylated proteins during physiological protein turnover (8). It inhibits nitric oxide synthase (NOS), an enzyme that catalyzes the conversion of L-arginine to nitric oxide (NO) and citrulline (8). As a result, a rise in ADMA lowers NO levels, a potent vasodilator essential for normal endothelial function. Impairments in the NO pathway leads to endothelial dysfunction, one of the earliest stages of initiation and progression of atherosclerosis $(8,9)$. In normal and atheromatous coronary arteries, NO plays an important role in acetylcholine-induced vasodilatation and resting vascular tone $(10,11)$. NO also inhibits platelet activation and aggregation (12).

In the past few years, AMDA emerged as a novel risk marker of CAD. Its levels were found to be elevated in risk factors contributing to $\mathrm{CAD}$ such as hypertension, type 2 diabetes mellitus, hypercholesterolemia and hyperhomocysteinemia. Further, all these conditions were associated with an impaired NO pathway and endothelial dysfunction (9). Studies have also linked ADMA to subclinical coronary atherosclerosis and adverse cardiovascular events $(13,14)$. The relationship between elevated ADMA and the angiographic extent of CAD has been studied by $\mathrm{Lu}$ et al and a few others (15-17). The aim of this study was to analyze serum ADMA levels in patients with varied extent and severity of coronary atherosclerosis and see if there were any major differences between male and females.

\section{Materials and Methods}

\section{Ethical committee approval}

This study was approved by the institutional ethics committee, Sri Ramachandra Institute of Higher Education and Research, Chennai. Participants were informed of the study details and then written informed consent was obtained from them.

\section{Study design}

This pilot study was conducted at Sri Ramachandra Institute of Higher Education and Research, Chennai, India on a total of 40 individuals (men and women) from the Cardiology department between the ages of 30 to 60 , with angiographically proven obstructive CAD. The participants medical history, personal history, family history and general physical characteristics were then collected and documented. According to their coronary angiographic reports, participants were divided into four groups: Group I - minor CAD, Group II - single vessel disease (SVD), Group III - double vessel disease (DVD) and Group IV - triple vessel disease (TVD).

\section{Laboratory analysis}

Venous blood samples were collected in gel tubes from the participants to estimate serum ADMA. The samples were centrifuged after 30 minutes at 1008 RCF. Next, the serum was separated, aliquoted into polypropylene tubes and then stored at $-20{ }^{\circ} \mathrm{C}$ until further analysis. Serum ADMA levels were estimated using the enzyme-linked immunoassay (ELISA) technique (Sincere Biotech Co Ltd, ELISA kit for measuring Human AMDA). Using the mean absorbance value for each sample, a standard curve was constructed and used to determine the corresponding concentration of ADMA was determined in $\mathrm{ng} / \mathrm{mL}$. The results were calculated using the ELISA reader software program.

\section{Statistical analysis}

For data analysis, we used SPSS software (version 18.0). We used the mean, standard deviation (SD) and p-values to compare serum ADMA levels among the four groups. We also calculated the continuous mean \pm SD. To evaluate the statistical significance, we conducted an independent sample t-test. We also compared ADMA levels between male and female participants. A p-value of less than 0.05 was considered statistically significant. 


\section{Results}

Table 1 shows ADMA level among the male ( $\mathrm{n}=$ $22)$ and female $(n=18)$ participants. Though the mean ADMA level among the male subjects was slightly lower than the females, there was no significant difference between them ( $\mathrm{p}$-value $=$ 0.534 ). The study participants were divided into four groups based on the extent and severity of CAD. Table 2 shows the comparison of ADMA levels between the minor CAD, SVD, DVD, and TVD groups. The values were found to be statistically significant ( $\mathrm{p}$-value< 0.0001 ). The results showed that Group II had a higher ADMA value than Group I, Group III had a higher ADMA value than Group II and Group IV had a higher ADMA value compared to Group III.

Table 1. Comparison of serum ADMA level between male and female.

\begin{tabular}{cccc}
\hline Parameter & Male & Female & p- value \\
\hline $\begin{array}{c}\text { ADMA } \\
(\mathrm{ng} / \mathrm{mL})\end{array}$ & $152.70 \pm 19.36$ & $156.49 \pm 14.22$ & 0.534 \\
\hline
\end{tabular}

p-value $<0.05$ is considered as statistically significant.

Table 1 depicts the mean and SD of the serum ADMA levels of the study participants. Among the study participants 22 were male and 18 were female. There is no statistically significant difference in the serum ADMA levels between the male and the female study participants $(\mathrm{p}=0.534)$.

Table 2. Serum ADMA levels of Study groups I, II, III and IV.

\begin{tabular}{ccccc}
\hline Parameter & Group I & Group II & Group III & Group IV \\
\hline $\begin{array}{c}\text { ADMA } \\
(\mathrm{ng} / \mathrm{mL})\end{array}$ & $144.54 \pm 24.16$ & $149.54 \pm 16.39$ & $159.46 \pm 10.40$ & $167.74 \pm 16.69$ \\
\hline
\end{tabular}

Values were expressed as mean \pm SD.

\section{Discussion}

Studies conducted among different groups across the world have shown that ADMA levels were significantly increased in patients diagnosed with CAD (18). Elevated ADMA levels were also shown to predict the incidence of future myocardial infraction (MI) attacks in previously diagnosed CAD patients (19). Results from studies conducted on patients with CAD, showed that all the major atherosclerotic risk factors such as dyslipidemia, diabetes mellitus, smoking and hypertension had abnormal endothelial vasculature $(20,21)$. Disturbing the endothelial vasculature contributes to atherosclerotic plaque formation since the vascular endothelium is involved in vascular tone maintenance, thrombi resistance, control of cellular adhesion, vessel wall inflammation and proliferation of smooth muscle cells, among others (22). ADMA inhibits NOS, which in turn decreases NO production. The NO pathway plays an important role in vascular endothelium dysfunction (12). Hence, measurement of ADMA in individuals who are at risk of developing CAD could help with early detection and severity predictions.

Among female and male participants, we found no significant differences between their ADMA levels. A similar result was obtained from the study conducted by Denve et al (24).

Here, ADMA levels were measured among angiographically proven CAD patients with varying disease severity. Based on the angiographic reports, ten individuals were assigned to each of the four groups: minor CAD $(<20 \%$ stenosis in the coronary artery), single vessel disease (SVD), double vessel disease (DVD) and triple vessel disease (TVD). ADMA was highest in the TVD group followed by DVD, SVD and minor CAD ( $\mathrm{p}$ value $<0.0001$ ). A similar result was obtained in the study conducted by Olga et al., which aimed to estimate the relationship between ADMA and angiographic indices of extent and 
severity of coronary atherosclerosis in CAD patients (23). There was a significant difference in ADMA levels in patients with SVD, DVD and TVD $(0.49 \pm 0.10 \mu \mathrm{mol} / \mathrm{L}$, $0.50 \pm 0.10 \mu \mathrm{mol} / \mathrm{L}, \quad 0.52 \pm 0.11 \mu \mathrm{mol} / \mathrm{L}) \quad(\mathrm{p}-$ value $<0.001$ ) (23). Furthermore, our results aligned with the results of from $\mathrm{Lu}$ et al (16). In this study, they subdivided CAD patients into two groups based on their percentage of coronary artery stenosis. Group 1 consisted of subjects with mild CAD $(<50 \%$ stenosis of major coronary arteries) and Group 2 consisted of subjects with significant CAD $(>50 \%$ stenosis). The ADMA level in Group 2 was significantly higher than Group $1(0.66 \pm 0.17$ $\mu \mathrm{mol} / \mathrm{L}$ vs $.0 .44 \pm 0.09 \mu \mathrm{mol} / \mathrm{L} ; \mathrm{p}-$ value $<$ 0.001) (14). Ultimately, ADMA level increases with CAD severity.

\section{References}

1. Mehta PK, Wei J, Wenger NK. Ischemic heart disease in women: A focus on risk factors. Trends Cardiovasc Med. 2015;25(2):140-151.

2. Elizabeth GN, Eugene B. A tale of coronary artery disease and myocardial infarction. N Engl $\mathbf{J}$ Med. 2012;366(1):54-63.

3. Sriharibabu M. Changing trends in the prevalence of coronary heart disease. Indian Heart J. 2016;68(4):445-446.

4. Mostafa C, Hassan A, Ali M, Esmaeel B, Salman S, Fatemeh H. Oxidative stress status and liver markers in coronary heart disease. Rep Biochem Mol Biol. 2019;8(1):49-55.

5. Abhinav Goyal, Salim Yusuf. The burden of cardiovascular disease in the Indian subcontinent. Indian J Med Res. 2006;124(3):235-44.

6. Nathan D Wong. Epidemiological studies of CHD and the evolution of preventive cardiology. Nat Rev Cardiol. 2014;11(5):276-89.

7. Ghaffar A, Reddy KS. Burden of noncommunicable disease in South Asia. BMJ. 2004;328(7443):807-810.

8. Karsen S, Boger RH. ADMA and oxidative stress are responsible for endothelial dysfunction in hyperhomocystinaemia: effects of Larginine and $\mathrm{B}$ vitamins. Cardiovascular Research. 2012;96(2):167-171.
We concluded that ADMA concentration in serum could be useful in identifying whether CAD correlates significantly to the extent and severity of coronary atherosclerosis. Thus, ADMA can be used as a prognostic marker due to its association with coronary atherosclerotic burden.

\section{Acknowledgements}

The authors would like to thank the Department of Biochemistry, Sri Ramachandra Institute of Higher Education and Research, Chennai for permitting our researchers to conduct this study. The authors declare that there is no conflict of interest regarding the publication of this manuscript.

9. Boger RH, Stefanie MB, Szuba A, Tsao PS, Chan JR, Tangphao O, Blaschke TF, et al. Asymmetric dimethylarginine (ADMA): A novel risk factor for endothelial dysfunction. Its role in hypercholesterolemia. Circulation. 1998;98(18):1842-47.

10. Magnus P, Beaglehole R. The real contribution of the major risk factors to the coronary epidemics: time to end the "only- 50\%" myth. Arch Intern Med. 2001;161(22):2657-60.

11. Ezzati M, Vander HS, Rodgers A, Lopez AD, Mathers CD, Murrary CJL. Estimates of global and regional health gains from reducing multiple major risk factors. Lancet. 2003;362(9380):27180.

12. Ali A, Mohammad S, Farnaz ZA, Hossein T, Saaeedh MM, Daryoush HA, Bahare F. The status of nitric oxide and its backup, Heme oxygenase 1, in Thromboangitis obliterans. Rep Biochem Mol Biol. 2018;6(2):197-202.

13. Schulze F, Lenzen H, Hanefeld C, Bartling A, Osterziel KJ, Goudeva L, et al. Asymmetric dimethylarginine is an independent risk factor for coronary heart disease: results from the multicenter Coronary Artery Risk Determination investigating the Influence of ADMA Concentration (CARDIAC) study. Am Heart J. 2006;152(3):493.e1-8. 
14. Miyazaki H, Matsuoka H, Cooke JP, Usui M, and Ueda S, Okuda S, et al. Endogenous nitric oxide synthase inhibitor: a novel marker of atherosclerosis. Circulation. 1999;99(9):1141-1146.

15. Zoccali C, Bode-Boger S, Mallamaci F, Benedetto F, Tripepi G, Malatino L, et al. Plasma concentration of asymmetrical dimethylarginine and mortality in patients with endstage renal disease: a prospective study. Lancet. 2001;358(9299):2113-2117.

16. Lu TM, Ding YA, Charng MJ, Lin SJ. Asymmetrical Dimethylarginine: A Novel Risk Factor for Coronary Artery Disease. Clin. Cardiol. 2003;26(10):458-64.

17. Lu TM, Chung MY, Lin MW, Hsu CP, Lin SJ. Plasma asymmetric dimethylarginine predicts death and major adverse cardiovascular events in individuals referred for coronary angiography. Int J Cardiol. 2011;153(2):135-40.

18. Boger RH. Asymmetric dimethylarginine (ADMA) and cardiovascular disease: insights from prospective clinical trials. Vasc Med. 2005;10 Suppl 1:S19-25.

19. Mirjana D, Sunica KD, Cabarkapa V, Eremic N. Biochemical markers of atherosclerosis. JMB. 2008;27(1):148-153.

20. Tse-Min L, Yu-An D, Shing-Jong L, WenShin L, Ho-Chang T. Plasma levels of asymmetrical dimethylarginine and adverse cardiovascular events after percutaneous coronary intervention. Eur Heart J. 2003;24(21):1912-1919. 21. Cooke JP. Does ADMA cause endothelial dysfunction. Arterioscler Thromb Vasc Biol. 2000;20(9):2032-7.

22. Vinod A, Michael B, Mohammed M, Guy W, James L, Raymond MA, et al. Asymmetric Dimethylarginine Causes Hypertension and Cardiac Dysfunction in Humans and Is Actively Metabolized by Dimethylarginine Dimethylaminohydrolase. Arterioscler Thromb Vasc Biol. 2003;23(8):1455-1459.

23. Olga K, Andrzej S, Alain G. Differential associations of angiographic extent and severity of coronary artery disease with asymmetric dimethylarginine but not insulin resistance in nondiabetic men with stable angina: a cross-sectional study. Cardiovasc Diabetol. 2013;12:145.

24. Deneva K, Lyudmila D, Evgenia A, Todorka ZT. Plasma ADMA level in healthy people. Folia Med (Plovdiv). 2011;53(1):28-33. 